Tropical Journal of Pharmaceutical Research March 2016; 15 (3): 623-629

ISSN: 1596-5996 (print); 1596-9827 (electronic)

(C) Pharmacotherapy Group, Faculty of Pharmacy, University of Benin, Benin City, 300001 Nigeria.

All rights reserved.

Available online at http://www.tjpr.org http://dx.doi.org/10.4314/tjpr.v15i3.26

Original Research Article

\title{
Profiling and Identification of the Metabolites of Evodiamine in Rats using Ultra-Performance Liquid Chromatography with Linear Ion Trap Orbitrap Mass Spectrometer
}

\author{
Fang Ping Wu, Yong Yang, Liang Hong Liu, Hong Pu, Ping Jin, Zu Fu Yao* and \\ Wei Cai* \\ Dong Pharmaceutical Research of Hunan Key Laboratory, Hunan University of Medicine, Huaihua Hunan 418000, China
}

*For correspondence: Email: 623965021@qq.com, 20120941161@bucm.edu.cn, Tel: +86 745 2381253; Fax: +86 745 2381210

Received: 21 October 2015

Revised accepted: 20 February 2016

\begin{abstract}
Purpose: To develop a highly sensitive and specific ultra-performance liquid chromatography with linear ion trap Orbitrap mass spectrometer (UPLC-LTQ-Orbitrap) method to profile and identify the metabolites of evodiamine in rats.

Methods: First, blood samples were collected after oral administration of evodiamine to rats (50 mg/kg). Next, the plasma samples were pretreated using a solid-phase extraction (SPE) method. Finally, all the samples were analyzed by ultra-performance liquid chromatography LTQ-Orbitrap mass spectrometry (UPLC-LTQ-Orbitrap) coupled with electrospray ionization source (ESI) in negative mode.

Results: A total of 7 metabolites (2 phase I and 4 phase II metabolites, including 4 new metabolites, viz, 10-hydroxyevodiamine sulfate, 10-hydroxyevodiamine sulfate, 10-hydroxyevodiamine glucuronide and 3-hydroxyevodiamine glucuronide) as well as the parent drug itself, were detected and identified based on accurate mass measurements, fragmentation patterns, and chromatographic retention times. The in vivo metabolic reactions of evodiamine in rats were hydroxylation, hydroxylation + sulfate conjugation, and hydroxylation + glucuronidation.

Conclusion: These results provide better understanding of the metabolism of evodiamine as well as strong indications of the effective forms of the drug in vivo.
\end{abstract}

Keywords: Evodiamine, Ultra-performance liquid chromatography with linear ion trap-Orbitrap, Hydroxyevodiamine sulfate, Hydroxyevodiamine glucuronide, Metabolites

Tropical Journal of Pharmaceutical Research is indexed by Science Citation Index (SciSearch), Scopus, International Pharmaceutical Abstract, Chemical Abstracts, Embase, Index Copernicus, EBSCO, African Index Medicus, JournalSeek, Journal Citation Reports/Science Edition, Directory of Open Access Journals (DOAJ), African Journal Online, Bioline International, Open-J-Gate and Pharmacy Abstracts

\section{INTRODUCTION}

Evodiamine belonging to indoloquinazoline alkaloids, is a major constituent isolated from the herbal medicine Evodia rutaecarpa (Wu-zhu-yu), which has been officially listed in Chinese Pharmacopoeia [1] and widely used in China for more than 2000 years. Pharmacological studies indicate that it possesses variety of anti- thrombotic, anti-inflammatory, uterotonic, thermoregulatory, anti-obesity, hypotensive, and vasodilatory effects [2-5].

Previous reports demonstrated that evodiamine had low oral bioavailability in rats [6,7], which suggested that evodiamine might undergo an extensive first-pass metabolism. Pharmacological studies have revealed the 
cytotoxicity of evodiamine might be related to the transformation of evodiamine into its 10hydroxyevodiamine or other metabolites [8]. However, so far as we are aware, the biotransformation of evodiamine has not been fully investigated due to the limitations of analytical techniques [8-10]. For instance, only two and five metabolites were detected and identified in vivo and in vitro, respectively [8-11]. In this respect, it is important to characterize its metabolism profile in vivo, which can help to further understand the mechanism of action of evodiamine.

During the past decade, liquid chromatography/electrospray tandem mass spectrometry was the main method for the structural characterization of drug metabolites in vivo and in vitro $[12,13]$. Ultra-high performance liquid chromatography coupled with highresolution mass spectrometer (UPLC-HRMS) such as UPLC-LTQ-Orbitrap significantly contributes to the characterization of drug metabolites due to its higher separation and resolution capacities in a shorter time [14,15].

The principal metabolic pathways of evodiamine in rats were proposed for the first time in this study. This study is also useful to understand the drug's pharmacological effects.<smiles>CN1c2ccccc2C(=O)N2CCc3c([nH]c4ccccc34)C21</smiles>

Figure 1: The structure of evodiamine

\section{EXPERIMENTAL}

\section{Chemicals and reagents}

Evodiamine was isolated from the $95 \%$ aqueous ethanolic extract of Euodiae Fructus in our laboratory. Its structure was identified by comparing their UV, MS, ${ }^{1} \mathrm{H}-\mathrm{NMR}$ and ${ }^{13} \mathrm{C}-\mathrm{NMR}$ data with it's in the literature. Its purity was $>98$ $\%$ by HPLC analysis. The structure is displayed in Figure 1. Grace PureTM SPE C18 phase extraction cartridges (200 mg/3 mL, $59 \mu \mathrm{m}, 70 \AA$ ) were purchased from Grace Davison Discovery ScienceTM (Deerfield, IL, USA). Ultra-pure water used throughout the experiment was freshly prepared using a Milli-Q water purification system (Millipore, Billerica, MA, USA).
Acetonitrile was of HPLC-grade (Fisher, NJ, USA). All other chemicals and reagents were of analytical grade and commercially available.

\section{Animals and drug administration}

Six male Sprague-Dawley rats (weighing 200 $250 \mathrm{~g}$ ) were supplied by Beijing Weitong Lihua Experimental Animals Company (Beijing, China) and housed in controlled environmental conditions (temperature, $24 \pm 2{ }^{\circ} \mathrm{C}$; relative humidity, $70 \pm 5 \%$ ) with free access to food and water in a week for acclimatization. The rats were randomly divided into two groups: Group A $(n=3)$, drug group for plasma; Group $B(n=3)$, control group for blank plasma. Before the experiment, all rats were fasted for $12 \mathrm{~h}$ and fed with water. Evodiamine was suspended in $0.5 \%$ carboxymethylcellulose sodium (CMC-Na) aqueous solution. The rats from group $A$ were anesthetized with ether at $2 \mathrm{~h}$ after oral administration of evodiamine at a dose of 100 $\mathrm{mg} / \mathrm{kg}$ body weight and then sacrificed by decapitation. The rats from group $\mathrm{B}$ were oral administrated with $0.5 \%$ CMC-Na aqueous solution in the same way. The blood were withdrawn in heparinized centrifuge tubes and centrifuged at $4000 \mathrm{rpm}$ for $10 \mathrm{~min}$ to obtain the plasma. All plasma samples were stored at -20 ${ }^{\circ} \mathrm{C}$ until other pretreatment and analysis. The animal experiments were carried out in accordance with the guidelines for Animal Experimentation of Hunan University of Medicine, and the protocols were approved by the Animal Biomedical Ethical Committee of Hunan University of Medicine (approval ref no. kj-dw-20150901-01) [16].

\section{Sample preparation}

All plasma samples were pretreated by a solidphase extraction (SPE). An SPE cartridge was pretreated with $5 \mathrm{~mL}$ of water, $5 \mathrm{~mL}$ of methanol and $5 \mathrm{~mL}$ of water, successively. $1 \mathrm{~mL}$ sample of plasma was loaded, and allowed to flow through the SPE cartridge with gravity. The SPE cartridge was washed with $5 \mathrm{~mL}$ of water and $5 \mathrm{~mL}$ of methanol, successively. The methanol eluate was collected and evaporated to dryness under $\mathrm{N}_{2}$ at room temperature. The residue was redissolved in $100 \mu \mathrm{L}$ of acetonitrile/water (10:90, $\mathrm{v} / \mathrm{v}$ ) and centrifuged at $12,000 \mathrm{rpm}$ at $4{ }^{\circ} \mathrm{C}$ for 30 min. A volume of $2 \mu \mathrm{L}$ supernatant was injected into UPLC-LTQ-Orbitrap MS for analysis.

\section{Instrumentation and conditions}

All LC/MS analyses were performed on a Finnigan LTQ/Orbitrap (Thermo Electron, Bremen, Germany) coupled to an ESI source 
(Thermo Electron, Bremen, Germany). A Waters ACQUITY BEH C18 column $(2.1 \times 100 \mathrm{~mm}$ i.d., $1.7 \mu \mathrm{m})$ was used for separation of the metabolites at room temperature and a flow rate of $0.3 \mathrm{~mL} / \mathrm{min}$. The mobile phase consisted of water (solvent A) and acetonitrile (solvent B) using a gradient elution as follows: $0-2 \mathrm{~min}, 5$ $\%$ B; 2 - 3 min, 5 - $10 \%$ B; 3 - 25 min, $10-35$ $\%$ B; $25-30$ min, $35-80 \%$ B; $30-35$ min, 80 $\%$ B; $35-36 \min , 80-5 \%$ B; $36-40 \min , 5 \%$ B.

Samples were analyzed in the negative mode with the tune method set as follows: sheath gas (nitrogen) flow rate of 30 arb, aux gas (nitrogen) flow rate of 5 arb, spray voltage of $4.0 \mathrm{kV}$, capillary temperature of $350{ }^{\circ} \mathrm{C}$, capillary voltage of $25 \mathrm{~V}$, tube lens voltage of $110 \mathrm{~V}$.

In the full scan experiment, resolution of the Orbitrap mass analyzer was set at 30,000 and with a mass range of $\mathrm{m} / \mathrm{z} 100-800$. Datadependent MS/MS scanning was performed to minimize total analytical time as it can trigger fragmentation spectra of target ions. The collision energy for collision induced dissociation (CID) was adjusted to $30 \%$ of maximum, and the isolation width of precursor ions was m/z 2.0 Da.

\section{Peak selections and data processing}

Thermo Xcaliber 2.1 workstation was used for the data acquisition and processing. In order to obtain as many fragment ions of the metabolites as possible, the peaks detected with intensity over 10,000 were selected for identifications. The chemical formulas for all parent ions of the selected peaks were calculated from the accurate mass using a formula predictor by setting the parameters as follows: $\mathrm{C}[0-30], \mathrm{H}$ [0 - 50], O [0 - 20], S [0 - 4], N [0 - 4], Cl [0 - 4], and ring double bond (RDB) equivalent value [0 -15]. Other elements such as $\mathrm{P}$ and $\mathrm{Br}$ were not considered because they are rarely present in the complex matrix.

\section{RESULTS}

\section{Fragmentation pathway of evodiamine}

In order to facilitate the structural identification of the metabolite, the $\mathrm{MS}^{\mathrm{n}}$ fragmentation pattern of evodiamine was investigated [17]. The parent ion showed a deprotonated ion $[\mathrm{M}-\mathrm{H}]^{-}$at $\mathrm{m} / \mathrm{z}$ 302.1296 (2.8 ppm, $\mathrm{C}_{19} \mathrm{H}_{16} \mathrm{ON}_{3}$ ) in negative mode. Fragmentation of the parent ion provided characteristic fragment ions at $\mathrm{m} / \mathrm{z} 169.0765$ (2.5 ppm, $\mathrm{C}_{11} \mathrm{H}_{9} \mathrm{~N}_{2}$ ) and $\mathrm{m} / \mathrm{z} 142.0658$ (4.7 ppm, $\mathrm{C}_{10} \mathrm{H}_{9} \mathrm{~N}$ ) by the loss of the moieties $\mathrm{C}_{8} \mathrm{H}_{7} \mathrm{ON}$ and
$\mathrm{C}_{8} \mathrm{H}_{7} \mathrm{ON}+\mathrm{CNH}$, respectively, which were useful information in metabolite identification. Besides, the fragment at $\mathrm{m} / \mathrm{z} 142$ can be formed by loss of $\mathrm{CNH}$ from the ion at $\mathrm{m} / \mathrm{z} 169$ in the $\mathrm{MS}^{3}$ spectra. The $\mathrm{MS}^{\mathrm{n}}$ spectrum of evodiamine was shown in Figure 2.

\section{Detection and structural elucidation of metabolites}

After comparison the high-resolution EIC (HREIC) of the drug samples with corresponding control samples, a total of 7 metabolites as well as the parent drug were detected and identified. The HREIC of drug sample is shown in Figure 3. The chromatographic and mass spectrometric data of the parent drug and its metabolites are shown in Table 1.

\section{Metabolite M0}

Metabolite M0 was unambiguously identified as evodiamine by comparing the retention time, accurate MS and MS/MS spectra with the authentic reference.

\section{Metabolites M1 and M2}

Metabolites M1 and M2 were eluted at 17.42 and $19.29 \mathrm{~min}$ with the quasi-molecular ions of $\mathrm{m} / \mathrm{z}$ 318.1248 (3.6 ppm, $\mathrm{C}_{19} \mathrm{H}_{16} \mathrm{O}_{2} \mathrm{~N}_{3}$ ) and $\mathrm{m} / \mathrm{z}$ 318.1245 (2.6 ppm, $\mathrm{C}_{19} \mathrm{H}_{16} \mathrm{O}_{2} \mathrm{~N}_{3}$ ), 16 Da more than that of evodiamine, indicating that it might be the product of mono-hydroxylated of the prototype drug. This ion at $\mathrm{m} / \mathrm{z} 185$ in $M 1 \mathrm{MS}^{2}$ spectra was found to be characterization for the presence of a hydroxyl group at one of the positions of the aromatic ring of the indole moiety. Based on these results, metabolite M1 were proposed to be mono-hydroxylated on the indole ring. This ion at $\mathrm{m} / \mathrm{z} 169$ in $M 2 M^{2}$ spectra belonged to the aromatic ring of the indole moiety, suggesting that metabolite $M 2$ were proposed to be no mono-hydroxylated on the indole ring. Previous reports [11] showed that the metabolites 10-hydroxyevodiamine and 3hydroxyevodiamine of evodiamine is easy to produce, therefore, M1 and M2 were tentatively identified as 10-hydroxyevodiamine and 3hydroxyevodiamine, respectively.

\section{Metabolites M3 and M4}

Metabolites M3 and M4, possessing the same deprotonated molecular ion $[\mathrm{M}-\mathrm{H}]^{-}$at $\mathrm{m} / \mathrm{z}$ 398.0813 (2.0 ppm, $\left.\mathrm{C}_{18} \mathrm{H}_{12} \mathrm{O}_{5} \mathrm{~N}_{3} \mathrm{~S}\right)$, were detected at 15.81 , and $16.44 \mathrm{~min}$, respectively. This fragment ion at $\mathrm{m} / \mathrm{z} 318$ was observed by 
Wu et al
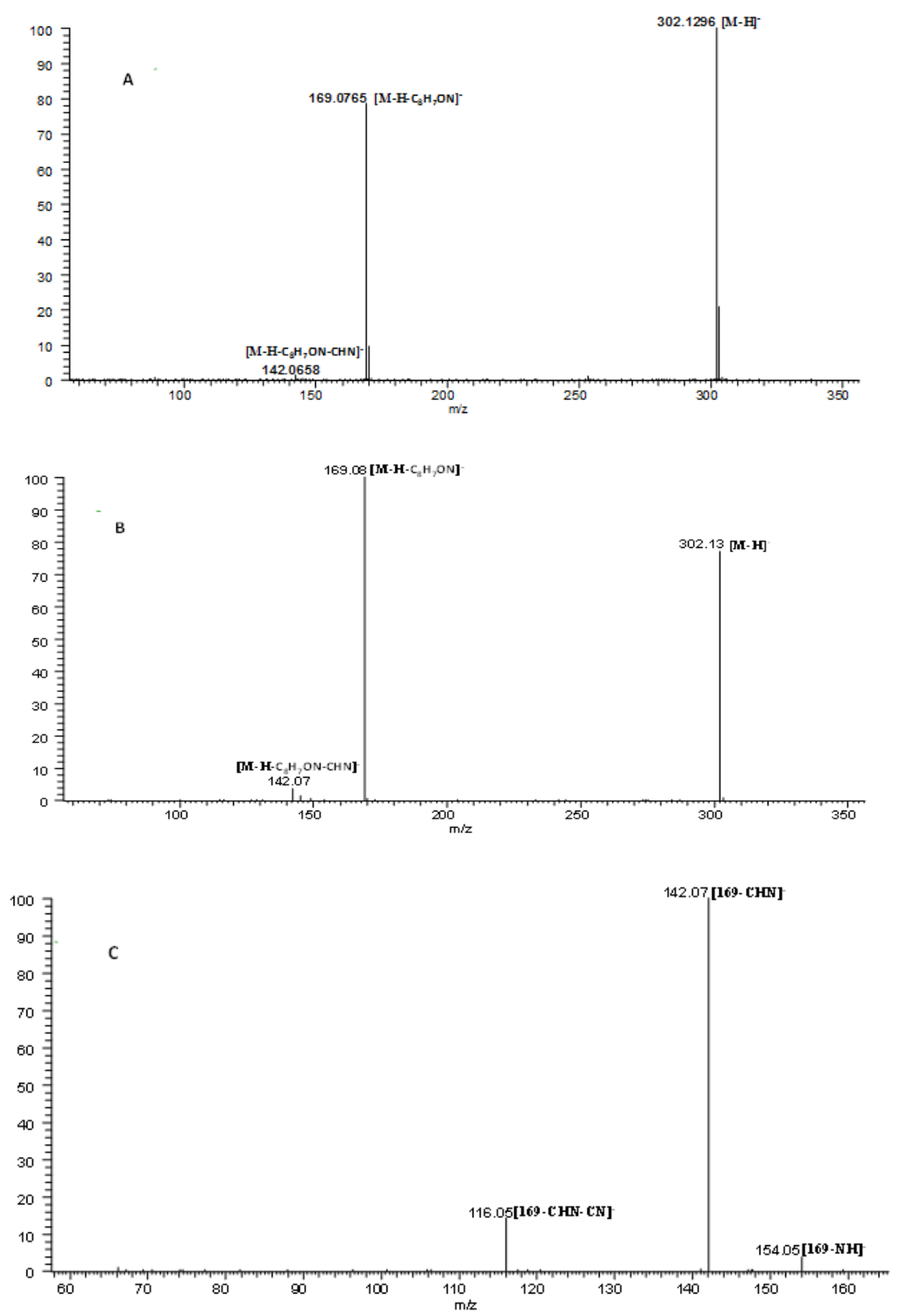

Figure 2: The $\mathrm{MS}^{\mathrm{n}}$ specturm of evodiamine

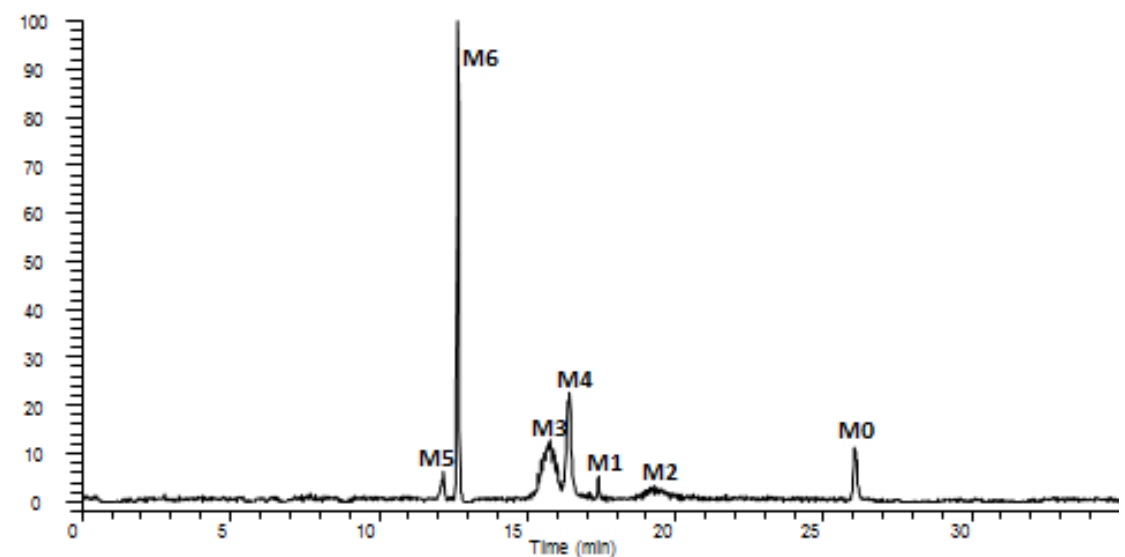

Figure 3: High-resolution EIC in 5 ppm for the multiple metabolites in rat plasma $\mathrm{m} / \mathrm{z} 302.1288,318.1237$, 398.0805, and 494.1558 
loss $80 \mathrm{Da}$ than the precursor ion at $\mathrm{m} / \mathrm{z} 398$ in their $\mathrm{MS}^{2}$ spectra, suggesting the presence of one sulfate residue in. The $\mathrm{MS}^{3}$ of M3 and M4 possessed the same $\mathrm{MS}^{2}$ spectra with $\mathrm{M} 1$ and $M 2$, respectively, suggesting the core structure of M3 and M4 were mono-hydroxylated of evodiamine. Therefore, M3 and M4 were tentatively characterized as 10hydroxyevodiamine sulfate and 3hydroxyevodiamine sulfate, which were new metabolites.

\section{Metabolites M5 and M6}

Metabolites M5 and M6 were eluted at 12.19, and $12.68 \mathrm{~min}$, with the quasi-molecular ions of $\mathrm{m} / \mathrm{z} 494.1573$ (3.2 ppm, $\mathrm{C}_{25} \mathrm{H}_{24} \mathrm{O}_{8} \mathrm{~N}_{3}$ ), and $\mathrm{m} / \mathrm{z}$ $494.1575 \quad$ (3.4 ppm, $\left.\quad \mathrm{C}_{25} \mathrm{H}_{24} \mathrm{O}_{8} \mathrm{~N}_{3}\right)$. This characteristic ion at $\mathrm{m} / \mathrm{z} 318$ was observed by loss $176 \mathrm{Da}$ than the precursor ion at $\mathrm{m} / \mathrm{z} 494$ in their $\mathrm{MS}^{2}$ spectra, which indicated that they might be products of glucuronide conjugation of $\mathrm{M} 1$ and M2. Analyzing the $\operatorname{cog} P$ values of their potential metabolites, M5 and M6 were tentatively assigned as 10-hydroxyevodiamine Sulfate and 3-hydroxyevodiamine sulfate, which were new metabolites.

\section{Proposed metabolic pathways of evodiamine}

In this study, 7 metabolites (4 new) as well as parent drug itself were detected in the plasma. The proposed major metabolic pathways of evodiamine in the rat plasma are demonstrated in Figure 4. In general, the metabolism of evodiamine in vivo was firstly undergone hydroxylation metabolic reactions (M1-M2), and then sulfate conjugation (M3-M4) and glucuronide conjugation (M5-M6) were taken place on the position of hydroxyl groups.

\section{DISCUSSION}

An UPLC-LTQ-Orbitrap mass spectrometry method for analysis the metabolites of evodiamine was established at first. In order to obtain an appropriate elution system, mobile systems such as acetonitrile - water and methanol - water were tested. The result demonstrated that acetonitrile - water with a gradient elution mode could afford better resolution of chromatographic peaks among the metabolites and endogenous components.

The evodiamine metabolites under investigation belong to indoloquinazoline alkaloids, which make it predestined detection by ESI in positive mode. However, Negative mode was selected for ESI-MS analysis in this study, as it provided more intensity response and less mass spectral interference [16]. To our best knowledge, the biotransformation of evodiamine has been poorly understood, although some works on the metabolism of evodiamine have already been performed [8-11]. For example, only one and two metabolites were unambiguously identified from

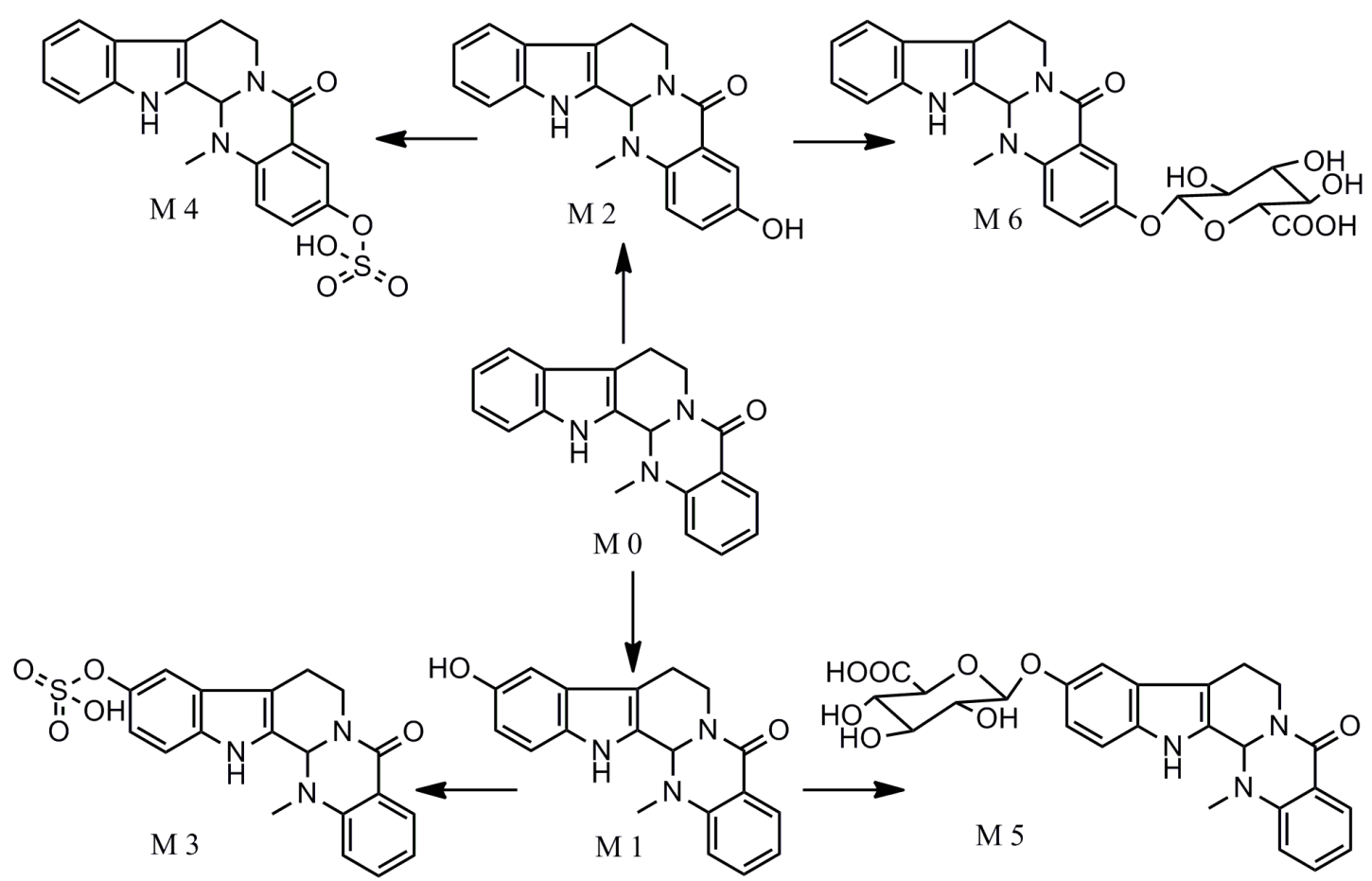

Figure 4: Proposed major metabolic pathway of evodiamine in rats 
Table 1: Details of evodiamine metabolites in rat plasma

\begin{tabular}{|c|c|c|c|c|c|c|c|}
\hline $\begin{array}{c}\text { Pea } \\
\mathbf{k}\end{array}$ & $t_{R}$ & $\begin{array}{c}\text { Theoretical mass } \\
(\mathrm{m} / \mathrm{z})\end{array}$ & $\begin{array}{c}\text { Experimental mass } \\
(m / z)\end{array}$ & $\begin{array}{l}\text { Error } \\
(\mathrm{ppm})\end{array}$ & $\begin{array}{c}\begin{array}{c}\text { Formula [M- } \\
\mathrm{H}]^{-}\end{array} \\
\end{array}$ & MS/MS fragment & Identification \\
\hline M0 & 26.05 & 302.1288 & 302.1296 & 2.8 & $\mathrm{C}_{19} \mathrm{H}_{16} \mathrm{ON}_{3}$ & $\begin{array}{l}M^{2}[302]: 169(100) \\
\text { 142(5) } \\
M^{3}[169]: \\
\text { 142(100),116(14),154(4) }\end{array}$ & evodiamine \\
\hline M1 & 17.42 & 318.1237 & 318.1248 & 3.6 & $\mathrm{C}_{19} \mathrm{H}_{16} \mathrm{O}_{2} \mathrm{~N}_{3}$ & $\begin{array}{l}M^{2}{ }^{2}[318]: 185(100) \\
158(15)\end{array}$ & 10-hydroxyevodiamine \\
\hline M2 & 19.29 & 318.1237 & 318.1245 & 2.6 & $\mathrm{C}_{19} \mathrm{H}_{16} \mathrm{O}_{2} \mathrm{~N}_{3}$ & $\begin{array}{l}M^{L^{2}}[318]: 169(100), \\
142(8) \\
M^{2}[398]: 318(100)\end{array}$ & 3-hydroxyevodiamine \\
\hline M3 & 15.81 & 398.0805 & 398.0813 & 2.0 & $\mathrm{C}_{19} \mathrm{H}_{16} \mathrm{O}_{5} \mathrm{~N}_{3} \mathrm{~S}$ & $\begin{array}{l}\mathrm{MS}^{3}[318]: 185(100) \\
158(8)\end{array}$ & 10-hydroxyevodiamine Sulfate \\
\hline M4 & 16.44 & 398.0805 & 398.0813 & 2.0 & $\mathrm{C}_{19} \mathrm{H}_{16} \mathrm{O}_{5} \mathrm{~N}_{3} \mathrm{~S}$ & $\begin{array}{l}\mathrm{MS}^{2}[398]: 318(100) \\
\mathrm{MS}^{3} \text { [318]: } 169(100)\end{array}$ & 3-hydroxyevodiamine Sulfate \\
\hline M5 & 12.19 & 494.1558 & 494.1575 & 3.4 & $\mathrm{C}_{25} \mathrm{H}_{24} \mathrm{O}_{8} \mathrm{~N}_{3}$ & $\begin{array}{l}\mathrm{MS}^{2}[494]: \\
318(100), 175(22)\end{array}$ & 10-hydroxyevodiamine Glucuronide \\
\hline M6 & 12.68 & 494.1558 & 494.1573 & 3.2 & $\mathrm{C}_{25} \mathrm{H}_{24} \mathrm{O}_{8} \mathrm{~N}_{3}$ & $\begin{array}{l}\operatorname{MS}^{2}[494]: \\
318(100), 175(42)\end{array}$ & 3-hydroxyevodiamine Glucuronide \\
\hline
\end{tabular}


urine and feces of SD rats by comparing the retention time and mass spectrometry of metabolites from Microbial biotransformation $[8,11]$. Five metabolites were tentatively detected and characterize in human liver microsomes. It is worth mentioning that none metabolites was detected in rat plasma including parent drug. In our study, 7 metabolites including parent drug itself were detected and identified in rat plasma. However, the previously reported metabolite named $\mathrm{N}$-demethylated evodiamine was undetected in both positive and negative MS modes in the present experiment, which might be because the species of experiment was different.

\section{CONCLUSION}

By online LC-MS ${ }^{n}$ data acquisition and offline data processing methods of the software Xcalibur 2.1, a total of 7 metabolites were detected and identified based on accurate mass measurements, the fragmentation patterns and chromatographic retention times. The findings show that the profile of the metabolites of evodiamine in rats can provide greater insight into its pharmacological effects, metabolic fate in vivo, and bioactive components.

\section{ACKNOWLEDGEMENT}

This work was supported by Science and Technology Innovation Team Project of Science and Technology Program in Huaihua (no. 20148) and Hunan University of Chinese Medicine High-level Talent Introduction Startup Funds (no. 15001).

\section{REFERENCES}

1. Chinese Pharmacopoeia Commission. Pharmacopoeia of the People's Republic of China, vol.1 China Medical Science Press, Beijing, China; 2010; $p 160$.

2. Chiou WF, Chou CJ, Shum AYC, Chen CH. The vasorelaxant effect of evodiamine in rat isolated mesenteric arteries: mode of action. Eur $\mathrm{J}$ Pharmacol 1992; 215(2): 277-283.

3. $Y u \quad H$, Jin $H$, Gong $W$, Wang $Z L$, Liang $H P$. Pharmacological actions of multi-target-directed evodiamine. Molecules 2013; 18(2): 1826-1843.

4. Tsai TH, Lee TF, Chen CF, Wang LCH. Thermoregulatory effects of alkaloids isolated from $\mathrm{Wu}$ chu-yu in afebrile and febrile rats. Pharmacol Biochem Behav 1995; 50(2): 293-298

5. Kobayashi Y, Nakano Y, Kizaki M, Hoshikuma K, Yokoo $Y$, Kamiya T. Capsaicin-like anti-obese activities of evodiamine from fruits of Evodia rutaecarpa, a vanilloid receptor agonist. Planta Med 2001; 67(7): 628-633

6. Komatsu K, Wakame K, Kano Y. Pharmacological properties of Galenical preparation. XVI. Pharmacokinetics of evodiamine and the metabolite in rats. Biol Pharm Bull 1993; 16(9): 935-938.

7. Shyr MH, Lin LC, Lin TY, Tsai TH. Determination and pharmacokinetics of evodiamine in the plasma and feces of conscious rats. Anal Chim Acta 2006; 558(1): 16-21.

8. Li L, Liu R, Ye M, Hu XY, Wang $Q$, Bi KX, Guo DA. Microbial metabolism of evodiamine by Penicillium janthinellum and its application for metabolite identification in rat urine. Enzyme Microb Technol 2006; 39(4): 561-567.

9. Jan WC, Lin LC, Don MJ, Chen CF, Tsai TH. Elimination of rutaecarpine and its metabolites in rat feces and urine measured by liquid chromatography. Biomed Chromatogr 2006; 20(11): 1163-1171.

10. Sun $H Z$, Fang ZZ, Cao $Y F$, Sun $X Y$, Hong $M$. Investigation of the in vitro metabolism of evodiamine: characterization of metabolites and involved cytochrome p450 isoforms. Phytother Res 2013; 27(5): 705-712.

11. Li L. Metabolic studies of ecodiamine, rutaecarpine and L-teterahydropalamatine in microbial organisms and rats. [Dissertation]. [Shenyang]: Shenyang Pharmaceutical University, 2005; pp 87-95.

12. Wani TA, Zargar S. New Highly-Sensitive UltraPerformance Liquid Chromatography-Mass Spectrometry Method for Quantification of Telmisartan in Human Plasma [J]. Trop J Pharm Res 2015, 14(3): 511-518.

13. Beaudry F, Yves Le Blanc JC, Coutu M, Ramier I, Moreau JP, Brown NK. Metabolite profiling study of propranolol in rat using LC/MS/MS analysis. Biomed Chromatogr 1999, 13(5): 363-369.

14. Zhang J, Cai W, Zhou Y, Liu Y, Wu XD, Li Y, Lu JQ, Qiao $Y J$. Profiling and identification of the metabolites of baicalin and study on their tissue distribution in rats by ultra-high-performance liquid chromatography with linear ion trap-Orbitrap mass spectrometer. $J$ Chromatogr B 2013; 985: 91-102

15. Ni SM, Qian DW, Duan JA, Guo JM, Shang EX, Shu $Y$, Xue CF. UPLC-QTOF/MS-based screening and identification of the constituents and their metabolites in rat plasma and urine after oral administration of Glechoma longituba extract. J Chromatogr B 2010, 878(28): 2741-2750.

16. Olfert ED, Cross BM, McWilliam AA. Guide to the care and use of experimental animals. Vol. 1. No. 2. Ottawa: Canadian Council on Animal Care, 1993.

17. Gao $P$, Wang LZ, Wu RG, Han J, Wang CY, Tang SM, Liu YG. Esi-ion-trp MS study on fragmentation pathways of evodiamine and rutecarpine. Chin J Pharm Anal 2012, 32(5): 772-774. 